\title{
Special issue (part II) on parallel computing for real-time image processing
}

\author{
Mohamed Akil $\cdot$ Laurent Perroton
}

Published online: 29 July 2011

(C) Springer-Verlag 2011

The performance requirements of image processing applications have continuously increased, especially when they are executed under real-time constraints. We have organized this special issue on Parallel Computing for Real-Time Image Processing to present the current state-of-the-art in the field of parallel programming and the future trends in realtime image and video processing as related to parallel computing or real-time implementation of embedded image processing applications on parallel architectures including multi-core platforms, graphics processors units (GPUs), and dedicated parallel architectures.

Due to the overwhelming number and their wide scope of submissions received for this special issue and thus the difficulty associated with finding expert reviewers, it was decided to offer this special issue in three parts as stated in the first part of this special issue. The papers that are currently under review will appear in the third part. We are very grateful to the reviewers who provided valuable comments and suggestions to improve the quality of the accepted papers.

This second part of this special issue on Parallel Computing for Real-Time Image Processing presents five papers addressing different parallel applications including 2-D digital curve approximation, object detection, Bayesian real-time perception, real-time medical video application and improvement of image quality. The real-time implementation of these applications uses GPU and FPGA platforms. Brief outlines of these papers are stated below:

\section{Akil $(\bowtie) \cdot$ L. Perroton}

Université Paris-Est, LIGM, Equipe A3SI, ESIEE Paris, Cité Descartes, BP 99, 93162 Noisy-le-Grand Cedex, France e-mail: akilm@esiee.fr

L. Perroton

e-mail: perrotol@esiee.fr
The first paper by Damiand and Coeurjolly presents a generic topological and geometrical framework, which allows defining and controlling several parallel algorithms for 2-D digital curve approximation. The proposed technique is based on combinatorial map simplifications guided by geometrical criteria. The genericity of the framework is illustrated by defining three contour simplification methods: a polygonal approximation one based on area deviation computation; a digital straight segments reconstruction, which guarantees to obtain a loss-less representation; and a moment preserving simplification, which simplifies the contours while preserving geometrical moments of the image regions. Thanks to a complete experimental evaluation, the authors demonstrate that the proposed technique can be efficiently implemented in a multi-thread environment to simplify labelled image contours.

The second paper by Herout, Jošth, Juránek, Havel, Hradiš and Zemčík presents the acceleration of object detection in images and video sequences using GPU. It includes algorithmic modifications and adjustments, constructing variants of efficient implementations and evaluation comparing to implementations on the CPU's. This article focuses on detection by statistical classifiers based on boosting. The implementation and the necessary algorithmic alterations are described, followed by experimental measurements of the created object detector and discussion of the results. The final solution outperforms the reference-efficient CPU/SSE implementation $\sim 6-8 \times$ for high-resolution videos using nVidia GeForce 9800GTX and Intel Core2 Duo E8200.

The third paper by Ferreira, Lobo and Dias presents the real-time implementation of a Bayesian framework for robotic multisensory perception on a graphics processing unit (GPU) using the compute unified device architecture 
(CUDA). In this paper, the authors show the benefits of parallel computing for similar problems (i.e., probabilistic grid-based frameworks), and the user-friendly nature of CUDA as a programming tool. They demonstrate that the SIMD (single-instruction, multiple-data) features of GPUs provide a means for taking a complicated framework of relatively simple and highly parallelisable algorithms operating on large data structures, which might take up to several minutes of execution with a regular CPU implementation, and arrive at an implementation that executes in the order of tenths of a second. The implemented multimodal perception module (including stereovision, binaural sensing and inertial sensing) builds an egocentric representation of occupancy and local motion, the Bayesian Volumetric Map (BVM), based on which gaze shift decisions are made to perform active exploration and reduce the entropy of the BVM.

The fourth paper by Savarimuthu, Kjaer-Nielsen and Sorensen presents an image processing involving correlation-based filter algorithm proven extremely useful for image enhancement, feature-extraction and recognition, in a wide range of medical applications, but it is almost exclusively used with still images due to the amount of computations required by the correlations. This paper presents two different practical methods for applying correlation-based algorithms to real-time video images using hardware-accelerated correlation, as well as the results in applying the method to optical venography. The first method employs a GPU-accelerated personal computer, while the second method employs an embedded-FPGA.

The fifth paper by Kurdthongmee proposes a hardware centric K-SOM quantizer algorithm, which relies on a rational-based representation of the codebook and learning kernel. This extends the capability of the quantizer to accept an approximated non-linear learning kernel. The experimental results indicate that the quality of the outcome images is superior to predecessor implementations with an acceptable throughput and FPGA resource utilizations. The results show that the image quality, measured by the mean square error (MSE), has an average improvement ratio of 0.68 compared with the state-of-the-art integer representation K-SOM quantizer for standard test images, whereas the proposed quantizer takes only $9 \%$ more of the FPGA resources.

\section{Author Biographies}

Mohamed Akil received his $\mathrm{PhD}$ degree from the Montpellier university (France) in 1981 and his doctorat d'état from the Pierre et Marie curie University (Paris, France) in 1985. He currently teaches and does research with the position of Professor at Computer Science Department, ESIEE, Paris. He is a member of Institut Gaspard-Monge, unité mixte de recherche CNRS-UMLPE-ESIEE, UMR 8049. His research interests are architecture for image processing, image compression, reconfigurable architecture and FPGA, high-level design methodology for multi-FPGA, mixed architecture (DSP/FPGA), system on chip (SoC) and parallel programming of 2-D/3-D topological operators. Dr. Akil has more than 80 research papers in the above areas.

Laurent Perroton is currently Associate Professor at the Computer Science Department, ESIEE, Paris. He is a member of the Insitut Gaspard Monge, unité mixte de recherche, CNRS-UMLPE-ESIEE, UMR8049. He completed his $\mathrm{PhD}$ thesis in 1995 on "Parallel 3D Segmentation" at the LIP, ENS Lyon, France. His main research interests are image processing and parallel computing. 